\title{
ASIC2a-dependent increase of ASIC3 surface expression enhances the sustained component of the currents
}

\author{
Hae-Jin Kweon ${ }^{1}$, Jin-Hwa Cho ${ }^{2}$, Il-Sung Jang ${ }^{2}$ \& Byung-Chang Suh ${ }^{1, *}$ \\ ${ }^{1}$ Department of Brain \& Cognitive Sciences, DGIST, Daegu 42988, ${ }^{2}$ Department of Pharmacology, School of Dentistry, Kyungpook \\ National University, Daegu 41940, Korea
}

\begin{abstract}
Acid-sensing ion channels (ASICs) are proton-gated cation channels widely expressed in the nervous system. Proton sensing by ASICs has been known to mediate pain, mechanosensation, taste transduction, learning and memory, and fear. In this study, we investigated the differential subcellular localization of ASIC2a and ASIC3 in heterologous expression systems. While ASIC2a targeted the cell surface itself, ASIC3 was mostly accumulated in the ER with partial expression in the plasma membrane. However, when ASIC3 was co-expressed with ASIC2a, its surface expression was markedly increased. By using bimolecular fluorescence complementation (BiFC) assay, we confirmed the heteromeric association between ASIC2a and ASIC3 subunits. In addition, we observed that the ASIC2a-dependent surface trafficking of ASIC3 remarkably enhanced the sustained component of the currents. Our study demonstrates that ASIC2a can increase the membrane conductance sensitivity to protons by facilitating the surface expression of ASIC3 through herteromeric assembly. [BMB Reports 2016; 49(10): 542-547]
\end{abstract}

\section{INTRODUCTION}

Acid-sensing ion channels (ASICs) are widely expressed in the central and peripheral nervous systems and mediate protonmediated neuronal signaling $(1,2)$. Tissue acidosis occurs during pathophysiological conditions, such as inflammation, tumorigenesis, and ischemic stroke (3-6). In addition, protons play roles as a neurotransmitter during normal brain activity (7). The perception of physiological $\mathrm{pH}$ change through ASICs mediates learning and memory, fear, taste transduction, mechanosensation, pain, and itching (3, 4, 8-12). ASICs are

${ }^{*}$ Corresponding author. Tel: +82-53-785-6123; Fax: +82-53-7856109; E-mail: bcsuh@ dgist.ac.kr

https://doi.org/10.5483/BMBRep.2016.49.10.057

Received 16 March 2016, Revised 8 April 2016, Accepted 27 May 2016

Keywords: Acid-sensing ion channel, Endoplasmic reticulum, Heteromeric assembly, Membrane protein, Surface trafficking voltage-independent and proton-activated cation channels that have high permeability to $\mathrm{Na}^{+}$ions (13). At least, six subunits (ASIC1a, ASIC1b, ASIC2a, ASIC2b, ASIC3, and ASIC4) are encoded by four genes (ACCN2, ACCN1, ACCN3, and $A C C N 4)$, and each subunit is composed of two transmembrane domains with a large extracellular loop and short cytoplasmic $\mathrm{N}$ - and C-termini. According to the crystal structure of ASIC1, three subunits assemble together to form a functional homo- or heteromeric channel (14).

Although the physiological roles of ASICs have been extensively studied, relatively little is known about the subcellular distribution and trafficking mechanisms of these channels. It is important that synthesized proteins are delivered to their cellular final destination. The surface composition and density of ion channels are tightly controlled by protein-sorting mechanisms (15). Synthesized proteins containing ER retention/retrieval signals or physiologically incorrectly assembled proteins are accumulated in the ER, and proper heteromeric assembly can antagonize ER protein retention (16). In some cases, properly assembled proteins require ER export signals for exiting from the ER (17).

According to previous studies, ASIC2a is predominantly expressed in the plasma membrane $(18,19)$. However, ASIC3 has shown a reticular pattern with a partial distribution in the plasma membrane when heterologously expressed $(20,21)$. In this study, we investigated the differential subcellular localization of these two subunits and found that ASIC2a plays an important role in facilitating the surface expression of ASIC3. In addition, we observed that the ASIC2a-dependent surface trafficking of ASIC3 markedly increased the sustained component of the currents. Our study has revealed a new trafficking mechanism of ASIC3.

\section{RESULTS AND DISCUSSION}

We first examined the subcellular distribution of ASIC2a and ASIC3 in human embryonic kidney (HEK) 293T cells. We fused GFP to the C-termini of ASIC proteins (ASIC2a-GFP and ASIC3-GFP, respectively) and transfected them into cells with a plasma membrane or an ER marker. As reported by the previous literature, we observed the substantial distribution of ASIC2a at the cell surface $(18,19)$. However, ASIC3 was 
mostly accumulated in the ER and around the nuclear envelope $(20,21)$. ASIC2a was highly overlapped with the plasma membrane marker, Lyn-mCherry (Fig. 1A and C). However, ASIC3 displayed a high overlap coefficient value with the ER marker, mCherry-Cb5 (Fig. 1B and C), consistent with the previous reports describing a reticular pattern of ASIC3 $(20,21)$.

We also examined their subcellular localization by Western blotting on cells expressing GFP-tagged ASIC2a or ASIC3. Immunoblotting with an anti-GFP antibody also revealed a high expression of ASIC2a in the plasma membrane (Fig. 1D). However, we observed a weak ASIC3 band of the predicted size in the plasma membrane fraction (Fig. 1D), suggesting that a portion of ASIC3 is also present at the cell surface, consistent with the previous study (21). The relative surface level of ASIC2a was about nine-fold higher than that of ASIC3 (Fig. 1D).

Previous studies have shown that several membrane receptors or ion channels are retained in the ER when heterologously expressed $(15,16)$. Such ER retention of synthesized proteins is generally caused by ER retention/ retrieval signals that mostly reside in $\mathrm{N}$ - or C-terminal regions of proteins. We investigated whether ASIC3 contains ER retention/retrieval signals in its cytoplasmic tails by constructing $\mathrm{N}$ - or C-terminal-deleted channels. However, the deletion of neither $\mathrm{N}$-terminal 2-43 amino acids (aa)

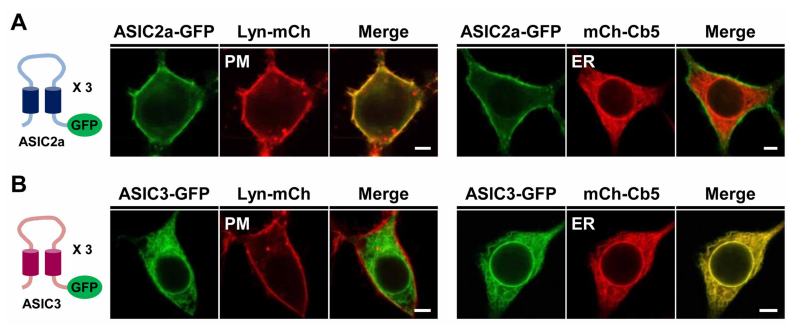

C

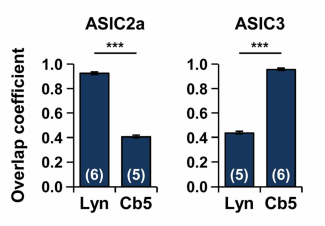

D

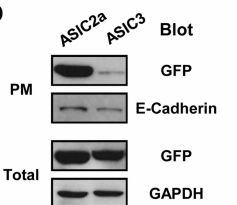

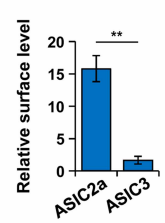

Fig. 1. Different subcellular distributions of $\mathrm{ASIC} 2 \mathrm{a}$ and $\mathrm{ASIC} 3$ in HEK293T cells. (A, B) Confocal images of HEK293T cells expressing GFP-tagged (A) ASIC2a or (B) ASIC3 with a plasma membrane (Lyn-mCh) or ER (mCh-Cb5) marker. Scale bars are 5 $\mu \mathrm{m}$. (C) Overlap coefficient value of fluorescent signals (mean \pm SEM, $* * * \mathrm{P}<0.001$, with Student's t-test). The number on each bar represents $\mathrm{n}$ for each condition. (D) Western blotting on the plasma membrane (PM) fraction and the total lysates of cells expressing GFP-tagged ASIC2a or ASIC3 using anti-GFP antibody. As controls, the PM and the total lysates were blotted using anti-E-Cadherin and anti-GAPDH antibodies, respectively. The surface level of ASIC2a or ASIC3 was normalized to that of E-Cadherin (mean \pm SEM, $n=3$ for ASIC2a; $n=3$ for ASIC3, $* * P<0.01$, with Student's t-test).
$(\operatorname{ASIC} 3(\Delta \mathrm{N}))$ nor $\mathrm{C}$-terminal $473-530$ aa $(\operatorname{ASIC} 3(\Delta \mathrm{C}))$ rescued ASIC3 from ER accumulation, suggesting that ASIC3 may not contain ER retention/retrieval signals in either the $\mathrm{N}$ - or C-terminal region we deleted (Supplementary Fig. S1). The deletion of both $\mathrm{N}$ - and C-termini from $\operatorname{ASIC3}(\operatorname{ASIC} 3(\Delta \mathrm{N}, \mathrm{C})$ ) was also ineffective for the localization of ASIC3 (Supplementary Fig. S1).

Recently, it has been reported that ASIC2a enhances the surface expression of ASIC1a $(22,23)$. To test whether ASIC2a can also deliver ASIC3 to the cell surface, we co-expressed ASIC2a and ASIC3 in HEK293T cells. As shown in Fig. 2A, ASIC3 successfully targeted the cell surface when it was co-expressed with ASIC2a. It was supported by a high overlap coefficient value between two subunits (Fig. 2B). We also examined the relative surface level of ASIC3 in the absence or presence of ASIC2a by Western blotting. As shown in Fig. 2C, the surface level of ASIC3 significantly increased in the presence of ASIC2a.

We then tested a heteromeric association between ASIC2a and ASIC3 by using bimolecular fluorescence complementation (BiFC) assay, which allows the visualization of protein-protein interactions in living cells (24). The C-termini of ASIC2a and ASIC3 were fused by one of the complementary fragments of the Venus protein, C-terminal half (VC) and

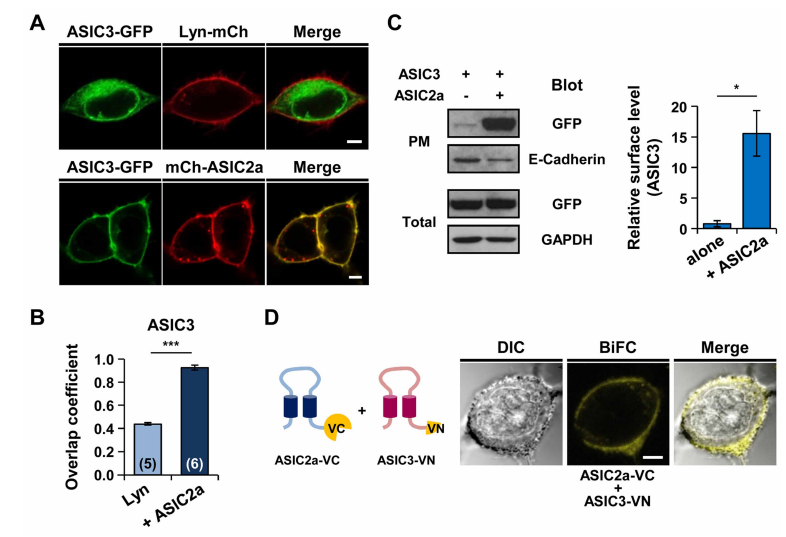

Fig. 2. ASIC2a enhances the surface expression of ASIC3 by heteromeric assembly. (A) Confocal images of HEK293T cells expressing GFP-tagged ASIC3 in the absence or presence of ASIC2a. (B) Overlap coefficient value of fluorescent signals (mean \pm SEM, with Student's t-test). Overlap coefficient value between ASIC3 and Lyn in Fig. 1C was plotted as a control (light blue). The number on each bar represents $n$ for each condition. (C) Western blotting on the plasma membrane (PM) fraction and the total lysates of cells expressing GFP-tagged ASIC3 in the absence or presence of ASIC2a in pcDNA3.1(+). As controls, the PM and the total lysates were blotted using anti-E-Cadherin and anti-GAPDH antibodies, respectively. The surface level of ASIC3 in the absence or presence of ASIC2a was normalized to that of $\mathrm{E}-$ Cadherin (mean \pm SEM, $\mathrm{n}=3$ for ASIC3 alone; $\mathrm{n}=3$ for ASIC3 with ASIC2a, ${ }^{* P}<0.05$, with Student's t-test). (D) Confocal images of HEK293T cells co-expressing ASIC2a-VC and ASIC3-VN. Scale bars are $5 \mu \mathrm{m}$. 
$\mathrm{N}$-terminal half $(\mathrm{VN})$, respectively. In cells co-expressing ASIC2a-VC and ASIC3-VN, we detected a BiFC signal in the plasma membrane, which was indicative of the heteromeric association between two subunits (Fig. 2D). However, we observed no fluorescence when either ASIC2a-VC or ASIC3-VN was expressed alone. These data suggest that ASIC2a delivers ASIC3 to the cell surface through a heteromeric assembly. However, when the $\mathrm{N}$ - or C-terminus was deleted or both the $\mathrm{N}$ - and C-termini were deleted, the ASIC2a-dependent surface trafficking of ASIC3 was significantly decreased (Supplementary Fig. S2), suggesting that both the $\mathrm{N}$ - and C-termini of ASIC3 are involved in the heteromeric assembly of ASIC2a and ASIC3 subunits.

We also performed electrophysiological experiments to investigate whether the ASIC2a-dependent surface trafficking of ASIC3 results in any alterations in ASIC currents. Rapid extracellular $\mathrm{pH}$ drop elicited inward currents both in cells expressing ASIC2a or ASIC3 (Fig. 3A). As previously reported, ASIC2a generated slowly activating and desensitizing currents, while ASIC3 elicited fast-activating and desensitizing transient currents followed by sustained currents (Fig. 3A). ASIC2a

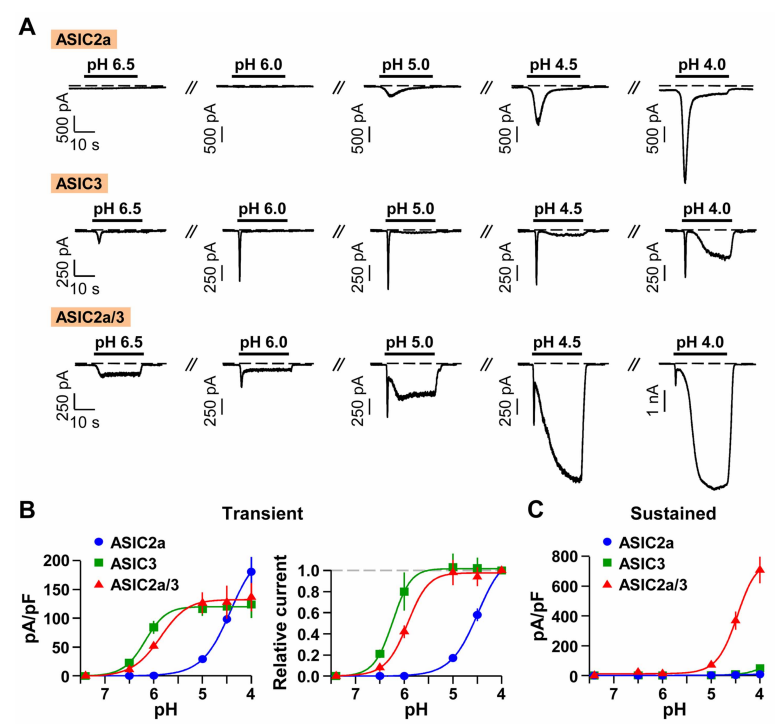

Fig. 3. ASIC2a-dependent surface trafficking of ASIC3 increases sustained currents. (A) Representative acid-evoked current traces of ASIC2a, ASIC3, or ASIC2a/3 channels. $\mathrm{pH}$ pulses were applied every $2 \mathrm{~min}$ for complete recovery from desensitization. Dashed line indicates the zero current level. (B) (left) pH-dependent current density of transient component of ASIC2a, ASIC3, or $\mathrm{ASIC} 2 \mathrm{a} / 3$ currents (mean $\pm \mathrm{SEM}, \mathrm{n}=5$ for ASIC2a; $\mathrm{n}=5$ for ASIC3; $\mathrm{n}=7$ for ASIC2a/3). (right) Relative current of ASIC2a, ASIC3, or ASIC2a/3 transient currents. Peak current at each $\mathrm{pH}$ was divided by $\mathrm{pH} 4$ 4.0-induced peak current in the left graph. (C) $\mathrm{pH}$-dependent current density of sustained component of ASIC2a, ASIC3, or ASIC2a/3 currents (mean \pm SEM, $n=5$ for ASIC2a; $\mathrm{n}=5$ for ASIC3; $\mathrm{n}=7$ for ASIC2a/3). The $\mathrm{pH}$-dependency curve was fitted with a Hill equation. generated distinct currents in response to an extracellular $\mathrm{pH}$ drop to 5.0 or more acidic $\mathrm{pH}$, while ASIC3 displayed higher sensitivity to protons; the half-maximal $\mathrm{pH}$ value $\left(\mathrm{pH}_{50}\right)$ of the ASIC3 transient currents was $6.1 \pm 0.2(n=5)$, while the $\mathrm{pH}$-dependency curve of ASIC2a did not reach a plateau even at $\mathrm{pH} 4.0$, as previously reported (Fig. 3B) (22). The sustained currents of ASIC3 were steadily increased by a decreasing extracellular $\mathrm{pH}$ value, and it was significantly increased when a pH pulse of 4.0 was applied (Fig. $3 \mathrm{~A}$ and $\mathrm{C}$ ).

In the ASIC2a/3 heteromeric channels, the current density of the transient component was not considerably altered compared with that of the ASIC3 homomeric channels. However, the $\mathrm{pH}$-dependent response curve was shifted to the intermediate between those of $\mathrm{ASIC} 2 \mathrm{a}$ and $\mathrm{ASIC} 3$; the $\mathrm{pH}_{50}$ value of the ASIC2a/3 transient currents was $5.8 \pm 0.1(n=7)$ (Fig. 3B). On the other hand, the sustained component of $\mathrm{ASIC} 2 \mathrm{a} / 3$ currents was remarkably increased compared with those of ASIC2a or ASIC3 homomeric currents (Fig. 3A and C). The current density of ASIC2a/3 sustained currents was about 14-fold higher than that of ASIC3 sustained currents at $\mathrm{pH} 4.0$ (Fig. 3C). Several studies have reported that heterologously expressed ASIC2a/3 channels generate robust currents (25-27). Our data suggest that an ASIC2a-dependent increase of ASIC3 surface expression may underlie these significantly enhanced sustained currents in ASIC2a/3 heteromeric channels.

Since ASIC2a and ASIC3 are co-expressed in several human tissues (27), we tested whether the ASIC2a-dependent trafficking of ASIC3 also occurred in neuroblastoma SH-SY5Y cells. As observed in HEK293T cells, ASIC2a and ASIC3 displayed differential subcellular localization. ASIC2a was primarily distributed at the cell surface, like RFP-PH (PLC $\delta)$, a plasma

A

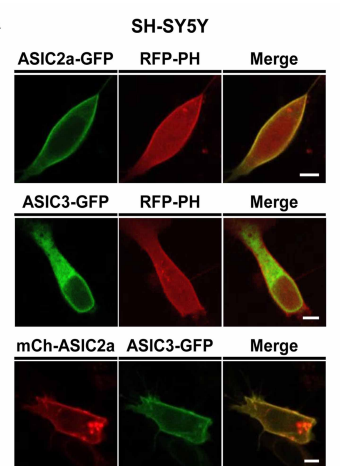

B

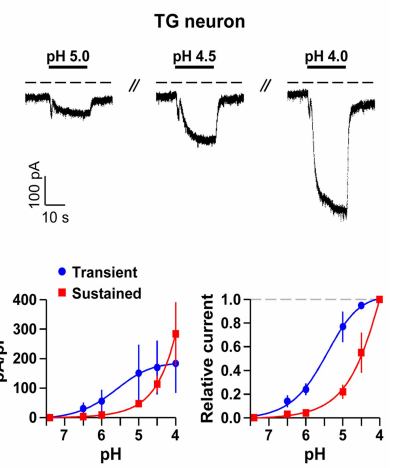

Fig. 4. ASIC2a-dependent surface expression of ASIC3 in SH-SY5Y cells and proton-induced currents in native neurons. (A) Confocal images of SH-SY5Y cells expressing GFP-tagged ASIC2a or ASIC3 with RFP-PH. Surface expression of ASIC3 is increased when it is co-expressed with ASIC2a. Scale bars are $5 \mu \mathrm{m}$. (B) Protonactivated currents in trigeminal ganglion (TG) neurons. (left) $\mathrm{pH}$-dependent current density (mean \pm SEM, $\mathrm{n}=4$ ). (right) Relative current. Peak current at each $\mathrm{pH}$ was divided by $\mathrm{pH}$ 4.0-induced peak current in the left graph. The pH-dependency curve was fitted with a Hill equation. 
membrane $\mathrm{PI}(4,5) \mathrm{P}_{2}$ probe (Fig. 4A). However, ASIC3 was accumulated in the ER and efficiently trafficked to the cell surface in the presence of ASIC2a (Fig. 4A). These results consistently demonstrate the significant role of ASIC2a in promoting the surface expression of ASIC3.

We also measured proton-induced currents in trigeminal ganglion (TG) neurons. According to the previous study, ASIC1, ASIC2a, and ASIC3 are highly expressed in TG neurons (28). In the middle-size (30-40 $\mu \mathrm{m}$ in diameter) neurons, we observed similar current patterns to those observed in HEK293T cells co-expressing ASIC2a and ASIC3. The sustained currents remarkably increased at $\mathrm{pH} 4.0$, and the $\mathrm{pH}_{50}$ value of the transient currents was $5.4 \pm 0.4(n=4)$ (Fig. 4B).

Lastly, we tested whether ASIC2b, a splicing variant of ASIC2a, can also deliver ASIC3 to the cell surface. ASIC2b has different amino acid sequences in the $\mathrm{N}$-terminus, the first transmembrane domain, and one third of the extracellular loop. Unlike ASIC2a, ASIC2b did not deliver ASIC3 to the cell surface (Supplementary Fig. S3B). ASIC2b was not even localized in the plasma membrane alone, as previously reported (29). To test whether the N-terminus of ASIC2a has a critical region for the membrane targeting of ASIC2a, we deleted 2-37 aa from $\mathrm{ASIC} 2 \mathrm{a}$. $\mathrm{ASIC} 2 \mathrm{a}(\Delta 37)$ mostly resided in the cytoplasmic area with ASIC3, and this mutated ASIC2a did not deliver ASIC3 to the cell surface (Supplementary Fig. S3C). For further analysis, we made more deleted ASIC2a. The deletion of 2-14 aa from ASIC2a had no effect on the membrane localization of ASIC2a and delivery of ASIC3 to the cell surface. However, the further deletion of ASIC2a disrupted the membrane localization of ASIC2a, and this $\operatorname{ASIC} 2 a(\Delta 30)$ could not deliver ASIC3 to the cell surface, suggesting that the critical region responsible for the membrane targeting of ASIC2a and for enhancing the surface expression of ASIC3 is located in 15-30 aa of ASIC2a.

In this study, for the first time, we report that ASIC3 trafficks to the cell surface in an ASIC2a-dependent manner. Since sustained currents are primarily mediated by $\mathrm{ASIC} 3$, the largely increased sustained currents in ASIC2a/3 heteromeric channels seem to be caused by the ASIC2a-dependent surface trafficking of ASIC3. According to the previous studies, the co-expression of ASIC2a and ASIC3 elicits proton-activated currents with changed biophysical properties (25-27). These alterations were recognized as an evidence for the functional interaction between two subunits (25-27). By using BiFC and the patch clamp technique, we found that the heteromeric association between ASIC2a and ASIC3 subunits forms proton-sensitive channels with remarkably enhanced sustained currents. We directly show another significant finding of the heteromerization of two subunits by revealing new trafficking mechanisms of ASICs.

\section{MATERIALS AND METHODS}

\section{Cell culture and transfection}

HEK293T and SH-SY5Y cells were maintained in DMEM supplemented with $10 \% \mathrm{FBS}$ and $0.2 \%$ penicillin/streptomycin at $37^{\circ} \mathrm{C}$ with $5 \% \mathrm{CO}_{2}$. The cells were plated in $35-\mathrm{mm}$ culture dishes at $50-60 \%$ confluency a day before transfection and transiently transfected using Lipofectamine 2000 (Invitrogen) according to the manufacturer's protocol. For the expression of heteromeric ASICs, we used each cDNA in a 1:1 molar ratio. The next day, the transfected cells were plated onto poly-Llysine $(0.1 \mathrm{mg} / \mathrm{ml}$, Sigma)-coated chips and studied 2 days after transfection.

\section{Trigeminal ganglion neuron preparation}

Sprague Dawley rats (5-6 weeks old, either sex) were decapitated under ketamine anesthesia $(100 \mathrm{mg} / \mathrm{kg}$, ip). Their trigeminal ganglia were dissected and treated with standard external solution (in $\mathrm{mM} ; 150 \mathrm{NaCl}, 3 \mathrm{KCl}, 2 \mathrm{CaCl}_{2}, 1 \mathrm{MgCl}_{2}$, 10 Glucose, and 10 HEPES, a pH of 7.4 with Tris-base) containing $0.3 \%$ collagenase (type I) and $0.3 \%$ trypsin (type I) for $30-40 \mathrm{~min}$ at $37^{\circ} \mathrm{C}$. Thereafter, the neurons were dissociated mechanically by trituration with fire-polished Pasteur pipettes in a culture dish. Isolated neurons were used for electrophysiological experiments 1-6 h after preparation.

\section{Plasmids}

Mouse cDNA clones of ASIC2a (GenBank accession no. NM 001034013.2) and ASIC3 (GenBank accession no. NM 183000.2) were generously given to us by Michael J. Welsh (University of lowa, lowa City, lowa). The cDNA encoding mouse ASIC2a was amplified by PCR and cloned into pEGFP-N1 (Clontech) using the EcoRI and BamHI sites or mCherry-C1 (Clontech) using the EcoRl and Kpnl sites. The cDNA-encoding mouse ASIC3 was amplified by PCR and cloned into pEGFP-N1 (Clontech) using the EcoRI and Kpnl sites. The plasmids Lyn-mCherry and mCherry-Cb5 were gifts from Takanari Inoue (Johns Hopkins University School of Medicine, Baltimore, Maryland).

\section{Molecular cloning}

For the generation of $\mathrm{N}$ - or C-terminal-deleted ASIC3, we used a QuikChange Site-Directed Mutagenesis kit (Agilent). The following primers were used for mutagenesis: sense, 5'-gggctgtgggccacagctgta-3', antisense, 5'-catgaattcgaagcttgagct cga-3' for $\operatorname{ASIC} 3(\Delta N)$ and sense, 5'-acggtaccgcgggcccgggat- 3 ', antisense, $5^{\prime}$-ccagaagtaccccaggactct- $3^{\prime}$ for $\operatorname{ASIC} 3(\Delta C)$. For the generation of $\mathrm{N}$ - and C-terminal-deleted ASIC3, CDNA encoding 44-472 aa of ASIC3 was amplified by PCR and inserted into pEGFP-N1 (Clontech) using the EcoRI and Kpnl sites. PCRs were carried out using high-fidelity DNA polymerase, and the products were verified by DNA sequencing (Macrogen). 


\section{Electrophysiology}

Whole-cell patch clamping was performed as described previously (30). For the acquisition and analysis of data, we used Pulse/Pulse Fit software in combination with an EPC-10 patch clamp amplifier (HEKA Elektronik). Further data processing was performed with Igor Pro (WaveMetrics, Inc.). For measuring the currents from the trigeminal ganglion neurons, we used an Axopatch 200B amplifier (Molecular Devices). The neurons were voltage-clamped at a holding potential of $-60 \mathrm{mV}$. Patch pipettes were made from borosilicate capillary glass (Narishige) by use of a pipette puller (Sutter Instrument). The resistance of the recording pipettes filled with the internal solution (in $\mathrm{mM} ; 140 \mathrm{CsF}, 10$ $\mathrm{CsCl}, 2$ EGTA, 2 ATP-Na 2 , and 10 HEPES with a pH adjusted to 7.2 with Tris-base) was $0.8-1.5 \mathrm{M} \Omega$. The membrane currents were filtered at $1 \mathrm{kHz}$, digitized at $4 \mathrm{kHz}$, and stored on a computer equipped with pCLAMP 10.3 (Molecular Devices). During recordings, $10-\mathrm{mV}$ hyperpolarizing step pulses (30 ms in duration) were periodically applied to monitor the access resistance, and the recordings were discontinued if the access resistance changed by more than $15 \%$. All the extracellular solutions were applied using the "Y-tube system" for rapid solution exchange (31).

\section{Plasma membrane fraction and Western blotting}

To isolate the plasma membrane fractions from the cells, we used a Plasma Membrane Protein Extraction kit (Abcam, ab65400) that yields high purity $(>90 \%)$ following the manufacturer's instructions. Western blotting was performed as previously described (32). Briefly, the protein samples were separated by SDS-PAGE using $10 \%$ gels. The separated proteins were transferred to polyvinylidene fluoride membranes and blotted with anti-GFP (Thermo Fisher Scientific; 4B10B2, 1:2,000), anti-E-Cadherin (NOVUS; NBP142793, 1:1,000), or anti-GAPDH (Cell Signaling; \#2118, 1:10,000) antibodies. After washing the blots, the proteins were visualized using an ECL detection system (Bio-Rad).

\section{BiFC assay}

For BiFC assay, ASIC2a and ASIC3 were cloned into bimolecular fluorescence complement (pBiFC)-VC155 and pBiFC-VN173 vectors, respectively, using the EcoRI and Kpnl sites. The HEK293T cells were co-transfected with cloned BiFC vectors, and Venus fluorescence signals were observed using a Carl Zeiss LSM 700 confocal microscope (Carl Zeiss) at room temperature.

\section{Confocal imaging}

Live cells were imaged 2 days after transfection on poly-L-lysine-coated chips with a Carl Zeiss LSM 700 confocal microscope (Carl Zeiss) at room temperature. The images were scanned with a $40 \times$ objective lens at $1024 \times 1024$ pixels using the digital zoom on a coverglass-bottom dish filled with the external solution (in $\mathrm{mM} ; 160 \mathrm{NaCl}, 5 \mathrm{KCl}, 1 \mathrm{MgCl}_{2}, 2$
$\mathrm{CaCl}_{2}$, and 10 HEPES, adjusted to $\mathrm{pH} 7.4$ with tetramethylammonium hydroxide).

\section{Quantitative analysis of fluorescent images}

Quantitative analysis of confocal images was carried out using ZEN2011 software (Carl Zeiss). The overlap coefficients of multiple sets of images were quantified by the following equation: Overlap coefficient $=\Sigma\left(\mathrm{Ch} 1_{\mathrm{i}}\right)\left(\mathrm{Ch} 2_{\mathrm{i}}\right) / \sqrt{(}\left(\Sigma\left(\mathrm{Ch} 1_{\mathrm{i}}\right)^{2}\left(\mathrm{Ch} 2_{\mathrm{i}}\right)^{2}\right)$, where $\mathrm{Ch} 1_{i}$ and $\mathrm{Ch} 2_{\mathrm{i}}$ denote the intensities of Channel 1 (GFP) and Channel 2 (mCherry), respectively. The overlap coefficient values are between 0 and 1 ; a value of 1 means complete co-localization, while a value of 0 means no co-localization occurs.

\section{Statistical analysis}

All quantitative data are represented as mean \pm SEM. Comparisons between two groups were analyzed using Student's two-tailed unpaired t-test and comparisons more than two groups were analyzed by one-way ANOVA followed by Bonferroni post-hoc test (33). Differences were considered significant at the $* \mathrm{P}<0.05$, ${ }^{*} \mathrm{P}<0.01$, and $* * * \mathrm{P}<0.001$ levels, as appropriate.

\section{ACKNOWLEDGEMENTS}

We thank the laboratories of Michael J. Welsh and Takanari Inoue for providing the plasmids. This work was supported by the DGIST R\&D Program of the Ministry of Science, ICT \& Future Planning (No. 16-BD-06), and the Korea Brain Research Institute basic research program funded by the Ministry of Science, ICT and Future Planning (No. 2231-415). The authors declare that no competing interests exist.

\section{REFERENCES}

1. Price MP, Snyder PM and Welsh MJ (1996) Cloning and expression of a novel human brain $\mathrm{Na}^{+}$channel. J Biol Chem 271, 7879-7882

2. Waldmann R, Champigny G, Bassilana F, Heurteaux C and Lazdunski M (1997) A proton-gated cation channel involved in acid-sensing. Nature 386, 173-177

3. Deval E, Noël J, Lay N et al (2008) ASIC3, a sensor of acidic and primary inflammatory pain. EMBO J 27, 3047-3055

4. Ikeuchi M, Kolker SJ, Burnes LA, Walder RY and Sluka KA (2008) Role of ASIC3 in the primary and secondary hyperalgesia produced by joint inflammation in mice. Pain 137, 662-669

5. Gupta SC, Singh R, Asters M et al (2016) Regulation of breast tumorigenesis through acid sensors. Oncogene In press

6. Smith ML, von Hanwehr R and Siesjö BK (1986) Changes in extra- and intracellular $\mathrm{pH}$ in the brain during and following ischemia in hyperglycemic and in moderately hypoglycemic rats. J Cereb Blood Flow Metab 6, 574-583

7. Du J, Reznikov LR, Price MP et al (2014) Protons are a 
neurotransmitter that regulates synaptic plasticity in the lateral amygdala. Proc Natl Acad Sci U S A 111, 8961-8966

8. Wemmie JA, Chen J, Askwith CC et al (2002) The acid-activated ion channel ASIC contributes to synaptic plasticity, learning, and memory. Neuron 34, 463-477

9. Wemmie JA, Askwith CC, Lamani E, Cassell MD Jr, Freeman JH and Welsh MJ (2003) Acid-sensing ion channel 1 is localized in brain regions with high synaptic density and contributes to fear conditioning. J Neurosci 23, 5496-5502

10. Ugawa S, Yamamoto T, Ueda T et al (2003) Amilorideinsensitive currents of the acid-sensing ion channel-2a (ASIC2a)/ASIC2b heteromeric sour-taste receptor channel. J Neurosci 23, 3616-3622

11. Chen CC and Wong CW (2013) Neurosensory mechanotransduction through acid-sensing ion channels. J Cell Mol Med 17, 337-349

12. Peng Z, Li WG, Huang $C$ et al (2015) ASIC3 mediates itch sensation in response to coincident stimulation by acid and nonproton ligand. Cell Rep 13, 387-398

13. Kweon HJ and Suh BC (2013) Acid-sensing ion channels (ASICs): therapeutic targets for neurological diseases and their regulation. BMB Rep 46, 295-304

14. Jasti J, Furukawa H, Gonzales EB and Gouaux E (2007) Structure of acid-sensing ion channel 1 at 1.9 A resolution and low $\mathrm{pH}$. Nature 449, 316-323

15. Ma D and Jan LY (2002) ER transport signals and trafficking of potassium channels and receptors. Curr Opin Neurobiol 12, 287-292

16. Michelsen K, Yuan H and Schwappach B (2005) Hide and run. Arginine-based endoplasmic-reticulum-sorting motifs in the assembly of heteromultimeric membrane proteins. EMBO Rep 6, 717-722

17. Ma D, Zerangue N, Lin YF et al (2001) Role of ER export signals in controlling surface potassium channel numbers. Science 291, 316-319

18. Chai S, Li M, Lan J, Xiong ZG, Saugstad JA and Simon RP (2007) A kinase-anchoring protein 150 and calcineurin are involved in regulation of acid-sensing ion channels ASIC1a and ASIC2a. J Biol Chem 282, 22668-22677

19. Chai S, Li M, Branigan D, Xiong ZG and Simon RP (2010) Activation of acid-sensing ion channel 1a (ASIC1a) by surface trafficking. J Biol Chem 285, 13002-13011

20. Hruska-Hageman AM, Benson CJ, Leonard AS, Price MP and Welsh MJ (2004) PSD-95 and Lin-7b interact with acid-sensing ion channel-3 and have opposite effects on $\mathrm{H}^{+}$-gated current. J Biol Chem 279, 46962-46968

21. Deval E, Friend V, Thirant C et al (2006) Regulation of sensory neuron-specific acid-sensing ion channel 3 by the adaptor protein $\mathrm{Na}^{+} / \mathrm{H}^{+}$exchanger regulatory factor-1. J Biol Chem 281, 1796-1807

22. Harding AM, Kusama N, Hattori T, Gautam M and Benson CJ (2014) ASIC2 subunits facilitate expression at the cell surface and confer regulation by PSD-95. PLoS One 9, e93797

23. Jiang $\mathrm{N}, \mathrm{Wu}$ J, Leng $\mathrm{T}$ et al (2016) Region specific contribution of ASIC2 to acidosis-and ischemia-induced neuronal injury. J Cereb Blood Flow Metab In press

24. Shyu YJ, Liu H, Deng X and Hu CD (2006) Identification of new fluorescent protein fragments for bimolecular fluorescence complementation analysis under physiological conditions. Biotechniques 40, 61-66

25. Hattori T, Chen J, Harding AM et al (2009) ASIC2a and ASIC3 heteromultimerize to form $\mathrm{pH}$-sensitive channels in mouse cardiac dorsal root ganglia neurons. Circ Res 105, 279-286

26. Hesselager M, Timmermann DB and Ahring PK (2004) pH Dependency and desensitization kinetics of heterologously expressed combinations of acid-sensing ion channel subunits. J Biol Chem 279, 11006-11015

27. Babinski K, Catarsi S, Biagini G and Séguéla P (2000) Mammalian ASIC2a and ASIC3 subunits co-assemble into heteromeric proton-gated channels sensitive to $\mathrm{Gd}^{3+}$. J Biol Chem 275, 28519-28525

28. Fu H, Fang $\mathrm{P}$, Zhou HY et al (2016) Acid-sensing ion channels in trigeminal ganglion neurons innervating the orofacial region contribute to orofacial inflammatory pain. Clin Exp Pharmacol Physiol 43, 193-202

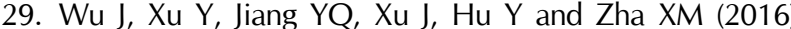
ASIC subunit ratio and differential surface trafficking in the brain. Mol Brain 9, 4

30. Kweon HJ, Yu SY, Kim DI and Suh BC (2015) Differential regulation of proton-sensitive ion channels by phospholipids: a comparative study between ASICs and TRPV1. PLoS One 10, e0122014

31. Murase K, Ryu PD and Randic M (1989) Excitatory and inhibitory amino acids and peptide-induced responses in acutely isolated rat spinal dorsal horn neurons. Neurosci Lett 103, 56-63

32. Lee HS, Lee GS, Kim SH, Kim HK, Suk DH, Lee DS (2014) Anti-oxidizing effect of the dichloromethane and hexane fractions from Orostachys japonicus in LPS-stimulated RAW 264.7 cells via upregulation of Nrf2 expression and activation of MAPK signaling pathway. BMB Rep 47, 98-103

33. Ko AR, Kang TC (2015) Mannitol induces selective astroglial death in the CA1 region of the rat hippocampus following status epilepticus. BMB Rep 48, 507-512 